\title{
Designing Windows for Interior Spaces for Thermal and Acoustic insulation
}

\author{
Assistant Professor Namer Kasim Khalaf, (Ph.D.) \\ College of Fine Arts \\ University of Diyala \\ Diyala - Iraq
}

\begin{abstract}
The Importance of this research (Design windows for Interior spaces for thermal and sound insulation), lies in the positive necessities to design windows for the internal spaces which must have high type designing features. This is done through using modern techniques and modern materials which are applicable and marketable and matches the Iraqi environment.

The Research aimed at:

- Designing windows for the Interior spaces with modern specifications and features (heat \& sound insulation).

- Describing the materials and techniques included in the design of glass and the building's windows.

The (Research Limitation) was limited to the subjective limit which is represented in the design of windows which isolate the heat and sound and the spatial limitations which are represented in the uses of these windows in designing the internal spaces for public and private buildings. Also, the current study was limited within its timing limitations on the date of designing the experimental study by the researcher in Year 2018. The Research Conclusions are:

- Using the Nano-Technology including (Nano-Ceramic) is to find amended and developed features for the production a new type of hard glass which is unbreakable where paresis occurs a consequence of presence of spaces between the atoms. This problem was overcome by reducing the spaces to the minimum which enabled using this technology in designing the windows in addition to increasing the heat and sound isolation property for the glass of these windows.

- Using various materials which are heat and sound insulators in various windows design joints, lead to an increase in the feature of the window deigned for heat and sound isolation.

The research reached the following important results:

- The designed sample is very effective for heat and sound isolation.

- Possibility of producing this type of windows to isolate some public and special internal spaces and the specialized spaces which need high heat and sound isolation.
\end{abstract}

Keywords: thermal insulation, acoustic insulation, interior spaces

\section{Introduction}

The provision of the internal physical environment suitable for the buildings users, are considered among the signs of civilization and among the features of the current era where we suffer from several problems in our internal environments (for our building which we use) where we agree upon creating buildings with functional spaces and with aesthetical specifications which carry rest and safety conditions with an integrated matrix of technical and complementary conditions. The buildings are considered as covers to internal spaces which serve the various humanitarian activities and that most the environmental problems in the buildings lie within the internal spaces. Here, comes the role of the internal designer in creating internal comfortable and isolated as much as possible from the environmental factors which influence the human comfort such as heat, sound and noise where these factors cause for human various health and psychological problems and hence the researcher started thinking to find solutions to get rid of some undesirable effects through one of the main internal design elements (Windows).

The Windows are the external openings which are considered among the main elements in designing the buildings from the functional, aesthetical \& environmental aspects, in specifying the designing and architectural features for the building. The window is a complicated multi-task system which is responsible for the selective communication between the inside and outside. Through the good design, this system allows the passage of the desired environmental influences, isolating the undesirable effects and the other designing features. Example of this is allowing for the external view and not ignoring the plastic influence for the windows glass esthetically. Due to the variation of elements which must be halted or allowed to penetrate the windows, therefore the design of windows became a complicated and advanced process. 
Hence, definite techniques were approved in the design of windows for the internal spaces which have a potential for heat and sound isolation. This new technique had several specialties which cooperate to design windows which have high efficiency functionally and esthetically. This aims at achieving the maximum degrees of comfort for the human in his internal environment in which he lives and works. For the sake of this, this research used the design of windows for the internal spaces which have heat and sound insulation.

The importance of the current research lies in the necessities to design window for the internal spaces which are characterized with common design features using modern techniques and materials which are capable to implement and marketing to satisfy the need of the local market for designed products which conforms to the Iraqi environment.

\section{Research Objectives:}

This research aimed at the following:

- Design windows for the internal spaces with modern specifications and features (heat $\&$ sound insulators).

- The materials and the techniques used in the design of the glass and the windows of the buildings are defined.

\section{Research Limitations}

The limitations of the current research were limited to the subjective limits which was represented in designing windows which heat and sound insulators;' and the special limitation which took the form in the use of these windows in designing the internal spaces for the public and private buildings. The current study was limited to the date of performing the experimental study by the researcher in year 2018 .

\section{Heat \& Sound Insulations for the Internal Spaces ${ }^{(1 *)}$}

With the advance of civilization and the increase of the modern means and equipment's in our lives; in addition to the climate changes in the world, then appeared in existence many environmental problems surrounding the humans. These problems concern the rise or lowering of temperatures and thenoisy pollutant which affects negatively in the life, health \& productivity of the individual. The heat, sound and noise are among the pollutants which cause various health obstacles for the humans where the level of heat and the high sound have a direct influence in the internal environment on their users. They also have influence on the building itself where it became from the design necessities isolating the internal environment from the undesirable influences and keeping it away from the internal spaces [1].

Controlling and overcoming these problems varies according to type of use where the process of selecting the used raw materials and ending the internal spaces, influence on controlling these problems. The raw materials vary in their potential to absorb and in its extent of reflecting of heat and sound. Therefore, the good internal design from the heat sound aspect, is linked with principles which must be available and which the designer must follow in the first stages of his work. The insulated materials can be divided in this research into materials and raw materials which are heat insulators and other sound and noise insulators where each have their own specifications.

\section{a) Materials \& Raw Materials of the Heat Insulation}

The available insulated materials vary in their potential on the heat conduction. For each material a fixed value concluded from the laboratory experiments which define amount of heat transfer in it. This value is called (Coefficient of Heat Conduction) where it is recommended to use materials with low capacity for heat conduction. The main concept is that the less the value of coefficient of heat conduction, then the heat gain or loss becomes less.

When designing the internal spaces, we should use (Porous Materials) for its good heat insulation where the selection of certain isolated material necessitates from the internal designer knowing its heat features in addition to other features such as water absorption and its ability to burn and its stiffness. Among the features of the heat isolated materials which can be employed in the internal design for the buildings or having heat and mechanical features plus the following features:

- The material potential to heat insulation, this potential is measured by the heat conduction coefficient where the lower coefficient of conduction, this implies an increase of the material resistance for transfer of heat and vice versa. The heat resistance is inversely proportional with the coefficient of heat conduction.

- Transfer of heat through the isolated material is usually done by all known means of transport which are (Conduction, Convection, Radiation). The reflecting materials are effective in the heat isolation for its high potential to repel the radiations and the heat waves provided it faces an air space.

\footnotetext{
(*) Review: The Researcher studies concerning the heat and sound insulation including: "Importance of Heat Insulation in designing the internal spaces for the residential buildings", Al-Fatih journal, Number (27), 2006 and "Using the sound insulation in designing the internal spaces for the large meetings halls", Al-Fatih journal, Number: 49, April 2012.

40
} 
- The ability of these materials increases by increasing its gloss where the isolated material is integrated with the walls and ceilings.

To know the total resistance for the heat transfer then we add its various external resistances including the resistance of the air layer touching the internal or external surfaces. Adding these resistances completely resembles adding the electrical resistances where they are either on parallel or successive where this depends on the position of the materials in the material surface.

- Some isolated materials are stiff and has a high potential on endurance. Therefore, we can use them in supporting and loading the building which is in addition to its main aim which is the heat isolation.

- The influence of humidity on the material depends on the properties of this material in terms of its ability to absorb and permeability. Also, depends on the surrounding climatic conditions which must be known for each isolated material [2].

\section{b) Materials for the Acoustic Isolation}

Most materials used in the internal design, absorbs the sound but they differences of the degree of absorbing the sound. There are materials with a high degree of absorbing the sound but there are other materials with little efficiency in absorbing the sound. Absorption of the sound means losing or dispersing it inside the space as the consequence of the vibrational energy for the sound waves and their transfer to dynamic or heat energy. The following specifications which are characterized by the sound insulation materials:

- The materials which are characterized by high degrees of absorption are either soft or porous or bending or a collection between more than any one of these features.

- To control the time of sound frequency for any internal space, and to reduce the level of noise resulted from the movement of the sound during the building factors, then we have to use materials with special specifications which are characterized with special characteristics with high coefficient for absorption of sound to limit its movement or return.

- In addition to the economic factor which plays an important role in using or not using those insulated materials but the price of the insulated material is expensive [2].

- There are other features which may be necessary when selecting the suitable isolated material such as knowing the density and the ability to resisting the shrinkage and possibility of being used several times ease of use, resisting the chemical interactions and the available measures and thicknesses. ${ }^{(*)}$

\section{Modern Techniques Used in the Heat and Sound Insulation in the Windows}

Many of the contemporary techniques which appeared recently can be used in the heat and sound insulation. Among the most modern techniques is the Nano, insulated glass techniques which is clarified as follows:

\section{Nano Technology and Paintings in the Heat \&Sound Insulation:}

This technology deals with measures which range between (10) to (100) Nanometers that is it deals with atomic conglomerates which range between (5) atoms to (1000) atom. This technology cares about the materials properties where the idea of using (Nano) techniques in re-ordering the materials atoms where whenever the atomic ordering of the material changes, then its resulted features changes. Here, the scientists managed to avoid some un-desirable features in some materials or the addition of other materials which double the performance efficiency of these materials. The (Nano-Technology) has low costs. It can manufacture the self-matching copies through placing systems which can copy itself and produces a useful product[3].

The (Nano-Technology) is used in painting in the glass or in the various designs and manufacturing materials which is concerned with the various internal spaces.

The scope of painting received so much research in the (Nano-Technology) where the idea of implementing the (Nanotechnology) in painting involves intensifying the chemical vapors to produce a layer linked with the main material to produce a surface containing the required protection.

The (Nano-Technology) is applied on the paintings and on the heat and sound insulated materials. Examples of these materials is a type of painting which consists of a mixture of the empty, spherical ceramic granules which works as the heat insulator. This painting is used on the internal and external walls where it reflects and disperses the sun ray because the ceramic painting is considered as a material for providing the heat energy where it has a double influence

\footnotetext{
${ }^{(* *)}$ Review : Al-Zaferani, Abbas Mohamed, "The Glass with the selective porosity for the sun radiation as an introduction to the environment design for the external openings in the buildings", Conference in Architectural Engineering Department, College of Engineering, Cairo University, 2006
} 
where it reflects and disperse the heat with its painting on the external walls. At the same time it keeps the heat of the internal space when it is used to paint the internal surfaces [3].(Figure (1) Appendixes List).

Other examples of the Ceramic Paintings is "ANZ" Paintings which form a layer with designed and developed specifications through using the (Nano-Technology). They consist of very accurate spherical crystals without spaces and are ordered in an engineering shape which works on the dispersion and reflection of the sun ray and its heat on the painted surface and consequently the internal temperature for the painted surface becomes lower than the natural temperature by more than (20) degrees centigrade.

When using the ceramic painting (ANZ), it doesn't require any external or internal paintings as an insulator to heat, sound, humidity and salts. The ceramic painting (ANZ-G) is used for glass to reduce the cost of electricity consumption by heat and sound insulation for the glass.

The ceramic painting ANZ contributes in achieving the sustainable internal design to be distinct, providing more than $20 \%$ from the used energy in the internal spaces in case of using it in windows glass painting or the glass walls where it works as a heat insulated layer.

The large economical influence appears in the locations which totally depend on the energy -where a one degree reduction in the cooling or heating temperature leads to a reduction of a $10 \%$ reduction of consumption of the electrical energy. By using ANZ Paintings, more than $80 \%$ of the ultra-red sun ray(NIR).

In General, the world started to link the (Nano) development with the sustainability in all the designs and projects for the importance of achieving the principles of humanitarian sustainability and the university where the (Nano) technique produces a new world from the advanced material which is more efficient and able to develop itself based on what is required by the designer. This gave new possibilities in the scope of sustainable internal design where it works on increasing the efficiency of the building performance and an improvement in the internal and external environment and the availability of energy consumption and the removal of the types of pollutant surrounding the humans. [4].

\section{Techniques in Using Glass in the Heat \& Sound Isolation}

Among the invented industrial materials which can be used as a heat insulator is the insulated glass where some of the heat leaks across the windows and doors glass. The rate of leakage is reduced by using the insulated glass in the windows and the glass doors where the energy consumption is reduced especially when using the glass which absorbs the heat. This type is used in the residential, commercial buildings, hospitals, restaurants and the buildings overlays the fast roads [5].

(Nano) materials added to the glass changes their properties and applications for example, Zink Oxide works with second titanium Oxide and nitrogen containing second titanium oxide to dispel the glass against the reflection of the sun ray [4].

Therefore, $\mathrm{TiO} 2$ is considered as a very important material used in the painting of the glass because the addition many of the properties to the glass such as resistance to pollution and water expulsion which makes the glass enjoys the feature of the self-cleaning. As for the $\mathrm{SiO} 2$ which is used as a painting layer between the layers of the glass where it works as a protection from the heat[6].

Some additions contributes to the glass from changing their properties which the contributions the additions from VO2Based by changing the color and heat. The additions works from NiO-Based and V2O5-Based change the color by the electrical wave the Nano Titanium Oxide works on the lighting self-cleaning[4].

These additions produced various types of glass. The following are the features characterized by the insulated and treated glass with the materials and the modern techniques:

- Prohibits the heat leakage of the sun ray across the windows which results in saving in the consumption of the energy using (thromocromatic) technology which is a very thin layer of paint which works on the heat insulation with the provision of the suitable lighting.

- Storing a certain amount of heat and then send it again inside the internal space in case of a reduction in the temperature so that it works as a warming equipment.

- Reflects the harmful sun ray ultra-violet.

- The feature of self-cleaning by analyzing the organic dirtiness and fog resistance.

- The technique of controlling in the changes of severity of lighting [3].

- Commercially, there are several types of glass (Figure: 1)

- Individual glass with one layer and various thicknesses.

- Glass bricks with two layers between which there is an air space for the heat insulation. 
- The insulated double glass which is provided with the heat mirror with two layers and between which there is an air space and a precise film called (Heat Mirror).

There are different types and names for the insulated glass types and therefore the internal designer must have a wide knowledge with these techniques and its precise needs and their suitability for the location where we want to design it. Examples of those several types and which are known in the scope of the internal design are: Ordinary glass, colored glass, reflector, reinforced, anti-lead (un-breakable), glass which doesn't allow the cohesion of the dust on its surface, anti-fire glass. Plus other types and with various thicknesses[7].

\section{The Insulated Materials Used in the Design of Windows:}

The designers of modern buildings stated that the windows are the weakest parts of the building which can disperse the different forms of data. This problem shows clearly in the modern large buildings in which area of the windows increases significantly.

To get rid of the heat insulation in case of the windows with ole glassy panel, intensive research were performed which concluded that the effective insulation which reduces significantly the expenditures of energy (Electricity, diesel, gas) is only achieved by using the double insulated glass where the units of the insulated double glass provides another benefit i.e. sound insulation.

The sound insulation is promoted significantly by setting up glass units with un-symmetrical thickness and by using a special gas in the space between the glass panels. The windows with double glass limits the loss of energy because the presence of the air space between the glass panels which is considered a poor conductor of heat and sound and that the selection of gases to fill the space between the double glass because a weaker conductor of heat from the air and hence the effectiveness of the window improves [8].

The insulated glass consists of two glassy panels which are parallel and fixed together to form a unit which is sealed with the rules. They are based on the principle which is forming a space between two or more glassy panels filled with dry air or another gas such as Argon or Xenon or Krypton. The Aluminum bar fills the space existing between the two panels with granules which absorb humidity.

When selecting the glass for the windows or doors or glassy facades where the following factors are considered:

- Ratio of the visual light which penetrates from the glass and it is preferred to be raised to reduce the electrical lighting and saving the consumption.

- Reduction in rate of penetration of the Alpha-red ray.

- Reduction in the (U-Value) which is the coefficient of heat transfer which is a measure of the scope of heat insulation for the dynamic heat energy as the consequence of variation if temperature between both sides of the glass.

- Reduction of the (Shading Coefficient) which is the ratio of the heat penetrating the glass compared with a transparent non-colored glass of (3) mm. thick.

When selecting the heat and sound insulators we should consider the general basis for selecting them and which takes into account the operational conditions concerned with the insulator and its ability to resist it and fulfilling the operational requirements required from it so that getting the best efficiency from the heat and sound insulators [8].

\section{Results and Discussion}

Designing Idea: Having inspected the available information stated in the theoretical framework concerning the heat and sound insulation materials which can be experimented on the design of a glassy window which can be used in isolating the internal spaces for the buildings. After inspecting the le commercial materials available in the local, Arab and international markets, the researcher reached a proposal for the design of glassy windows which can be insulated to heat and sound and has modified and developed features. By using techniques consisting of heat and sound insulated techniques and the Nano-Technology techniques in the painting materials for the glass and to increase the insulation feature for the glassy window. The researcher reached a proposed design for a window which can be produced and marketed and according the following design steps: (Figure (1) Appendixes List).

\section{Proposed Design:}

a) Design a Sample of the Window which insulate Heat \& Sound:

- The Window manufactured from $(\mathrm{PVC})^{(* * * 3 *)}$ material which is heat and sound insulated and treated by NanoTechnology.

${ }^{(* * * *)}$ This is a chemical polymeric material made of heat plastic with cohesive, white and solid color and has many uses such as heat and sound insulation. Review (Kobeily, 2009: Page: 20). 
- The Glass layers contain an insulated material of the glass perimeter manufactured using rubber.

b) Design a Sample of Glassy Panels Used in the Window:

- The window which insulates the heat and sound is described as a window with double glass because it contains threelayer glass which contains two spaces between the external and internal glass layer.

- The area between the glass layers contain an alert gas (The internal first contains Argon gas whereas the second layer contains Xenon gas. These gases have good insulation features.

- Polishing the outside glass area with a transparent (Nano-Ceramic) film which is insulated to heat and sound.

- The Glass thickness for the external and internal layers adds strength and better insulation capabilities resulted from the higher the thickness of glass but the window's weight becomes heavier. This matter is specified by the internal designer.

- Windows measurements depend on the type of space or the wall in which a window must be constructed.

c) Inspecting the Sample of Proposed Design:

The proposed design sample and the used raw materials are presented to specialized experts in the scope of design and the specialty of materials engineering and physics science and Nano-Technology. They provided a number of remarks concerning the type of used raw materials and method of using them in the proposed design including the type of (pvc) material available in the local market and how to treat its surfaces with Nano-Technology and the type of Rubber Silicon and its quantity. This is in addition to thickness of the used glass and types of inert gases and their availability in the market and possibility of their periodical maintenance and not leaked through the glass and the environmental resistance. This is in addition to size of window and their place of existence inside the buildings and lowering their costs. After considering the experts proposals, the design was amended reaching the final design pattern as shown in Figure (2)

The researcher designed a primary sample for the shape of the window designed according to the specifications stated in the theoretical framework and the designing idea and the proposed sample after amendment. Then calculating the heat and sound insulation coefficient compared with the ordinary glassy windows used in the internal spaces. Then performing a comparison with the insulated windows in the international market. After measuring the heat and sound insulation coefficient, the following results were reached:

- The designed sample is very effective in the heat and sound insulation.

- Possibility of producing this type of windows to insulate some internal spaces which need a high heat and sound insulation.

- Possibility of marketing it in the local market.

\section{Conclusions}

- Using the Nano-Technology including Nano-Ceramics Material, is find amended and developed features o produce a new type of solid glass which is un-breakable where the brittleness occurs a consequence of existence of spaces between the atoms. This was overcome by reducing the spaces between the atoms to reach its minimum. This enabled using this technique in designing the windows which is in addition to increasing the feature of the heat and sound insulation for the glass of these windows.

- Using different materials for heat and sound insulation in various windows designs joints, lead to an increase in the feature of the window designed for heat and sound insulation.

- Modern materials have characteristics which satisfy the desires of the designers and consumers which satisfy everybody from the functional, aesthetical \& economic aspects including using (PVC) materials in the production of windows and types of alert gases.

- The internal design departments students started to perform designing studies to overcome the internal spaces according to the development of materials and techniques for producing those including different Nano-Technology materials.

- Performing future scientific research concerning the uses of Nano-Technology in the various arts specialties.

\section{References}

Corky Binggeli, "Building Systems for Interior Designers", John Wily \& Sons, INC.2003,P91-92

Khalaf, Namir Kasim, "Techniques \& Complements of the Internal Design", Dar Safhat for Studies \& Publications, United Arab Emirates, Dubai, 2017,P141-214.

Lamis Sayed Mohamady, "Role of Technology in developing the Traditional Architectural Factors", (Master Thesis, Un-Published), College of Engineering, Alexandria University, 2011,P124-166. 
Horba, Ola , "Architecture in the shadow of Nano-Technology". College of Architecture, Volume:39, 2017,P98-99.

Al-Aloush , Reba, "Analytical Study for the features of the glassy walls - International \& Local Examples", Al-Jamea Magazine, Volume:36.P177.

Gomaa , Hussein Mohamed, "Encyclopedia of Paintings and Architectural and Industrial", Office of the Studies and the Engineering Consultancies, Egypt, 2004,P243-244.

Al-Zafrany, Abbas Mohamed, "The Selective Porosity Glass for the sun radiation principle for the environmental design for the external openings in the buildings", Conference, Architectural Engineering Department, College of Engineering, Cairo University, 2006.

Ali, Asad Hassan, Gorge, "Modern Materials in the Internal Cladding - Reality \& Horizons", Damascus University Magazine for the Engineering Sciences, Volume 25.P20.

Kbeily,Yamin Ali, "Rationalization for the Energy Consumption by Improving specifications of some Heat Insulation Materials", (Un-Published Master Thesis). Mechanical Forces Engineering Department, Tishreen University, 2009,P13-18.

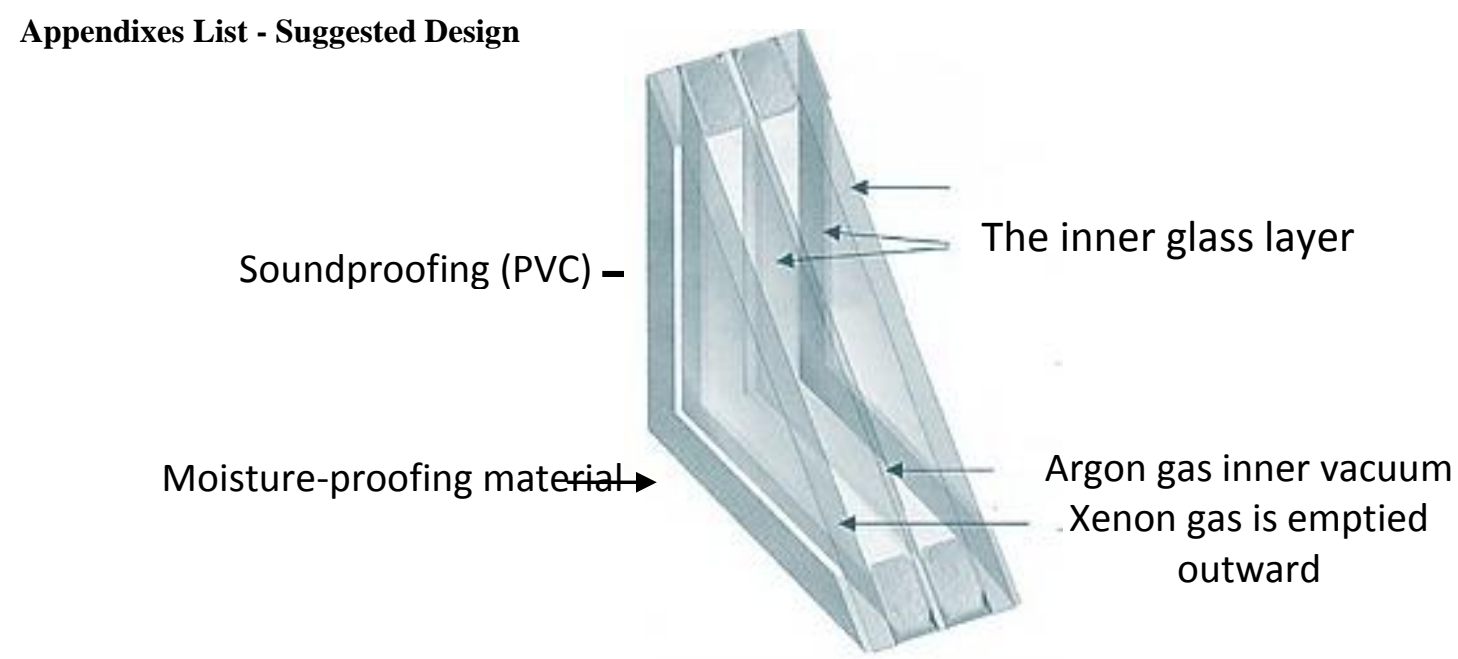

Figure (1)Processing layer Through nano-ceramics -Transparent

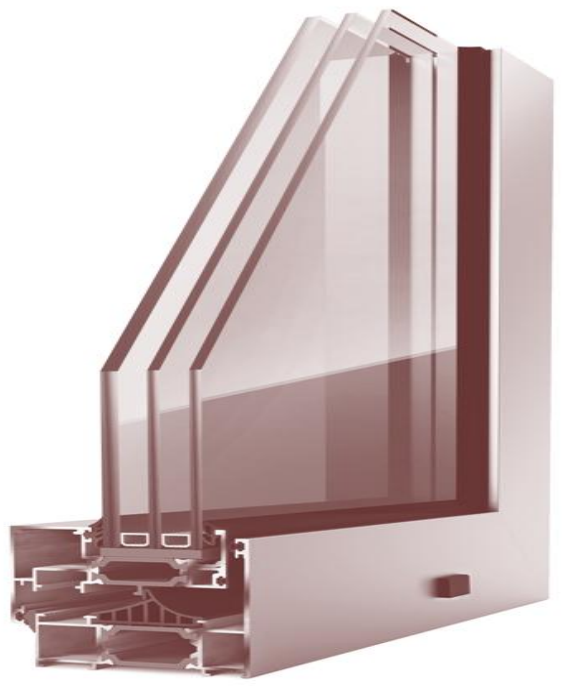

The shape of the final window

frame and nanotechnology

processor

Figure (2)The design proposed by the researcher for sound and heat insulation window 